\title{
Feuilleton.
}

\section{Zur Pathogenese der Geistes- und Nervenkrankheiten im Orient.}

Von Massar Bey, Dozenten der Neurologie am GullhaneLehrkrankenhause in Stambul (Oberarzt Raschid Tahsin Bey).

Es wird in einschlägigen Werken des Auslandes-häufig aus geführt, daß Geisteskrankheiten im Orient selten seien, und man gibt als Ursache eine gewisse Immunität des Klimas an. Es nehmen aber die Geisteskrankheiten auch bei uns zweifellos zu, ohne freilich die Höhe der westeuropäischen Länder zu erreichen. Suchen wir nach der Ursache, indem wir Vergleiche zwischen Westeuropa und unserem Lande ziehen!

Ein Hauptfaktor für Erkrankungen des Nervensystems sind Intoxikationen. Professionelle Bleivergiftungen, die für Polyneuritis, aber auch für Psychosen verschiedener Art in Betracht kommen, sind selten. Morphium, Kokain, Atropin sind im Volke völlig unbekannt. Sehr selten begegnet man in den Asylen einem Opiomanen oder Haschischomanen. Vor allem fehlt bei uns die so vierderbliche Wirkung des Alkohols, der den Grund für ungeheuer viele geistige und nervöse Erkrankungen abgibt. Dẹ Alkoholgenuß ist im Orient sehr beschränkt, als Laster kaum nennenswert verbreitet, auch die nicht muselmanische Bevölkerung ist weit mäßiger als die westeuropäischen Völker. So sind die Erkrankungen, die auf Alkoholmißbrauch zurückzuführen sind, Seltenheiten. Das Nervensystem der Orientalen ist weder individuell noch hereditär durch seine toxischen Einflüsse geschädigt.

Die geistige Beschäftigung ist im Orient sicher nicht so intensiv als im Westen, wenngleich die Anforderungen der vordringenden Kultur auch hierin Aenderungen ergeben werden und schon ergeben haben. Mehr als die geistige Ueberanstrengung kommen für Geisteskrankheiten indes wohl die tieferen Einwirkungen auf das Gemüts- und Seelenleben in Betracht, und diese sind im Orient minder intensiv. Die Religion und die Landessitten erleichtern dem Orientalen z. B. die Trauer und den Kummer um eine dahingeschiedene teure Person oder um den Verlust eines kostbaren Gutes: Wir versuchen den Verlust leicht zu vergessen, den wir mit unserer menschlichen Kraft doch nicht ändern können. Unsere Erziehung erlaubt uns nicht die völlige Hingabe an eine Person oder Sache, die mit der Zeit vergeht, und wir versucheil, uns mit diesem Gedanken vertraut zu machen, ehe wir sie in Wirklichkeit verlieren. Wir streben in dieser unserer Geistestätigkeit nach einem dauernden und logischen Ideal. Unsere ganze Existenz ist doch einem Zufall zu danken; was wir besitzen, bereitet uns nicht die höchste Freude, weil wir denken, daß es ja nicht unser Gut ist, und der Verlust dieses Gutes versetzt uns nicht in untröstliche Trauer. Wir arbeiten in der Hoffnung auf Gelingen wie als eine Pflicht; wenn es vergeblich ist, ist es nicht unsere Schuld, es lag in der Hand der großen unbesiegbaren Macht.

Ein weiterer Vorteil für die Diätetik des Nervensystems im Orient liegt in der Einfachheit und Natürlichkeit der Lebensweise. Abendgesellschaften, Bälle, Varietétheater im Sinne des Abendlandes gibt es bei uns kaum. Uebermaß im Genießen jeglicher Art verabscheut der Orientale. Er sucht sein Lebensglück im Familienleben. Er verheiratet sich früh, ohne vorher in den Abgründen der Unmoralität erschöpft oder gealtert zu sein. Ehescheidurgen sind leicht. Die Heirat ist kein Endziel für sie. -

Eine toxische Erkrankung, die bei uns sehr verbreitet ist und viel Schaden tut, ist der Paludismus. Die auf Malaria beruhenden Psychosen, die besonders als Melancholie zutage treten, geben oft zu falschen Diagnosen Anlaß. Die so häufigen, impulsiven Verbrechen bei uns lassen bisweilen an ein Malariaäquivalent denken, wenn man so sagen darf. Folgende zwei Beispiele mögen das belegen:

Ein Beamter der drahtlosen Telegraphie, der in malariareicher Gegend lebte, wurde im AnschluB an einen Trauerfall von Verfolgungswahnsinn ergriffen. Er hatte Halluzinationen (charakteristisches Stimmenhören, Gesichtstäuschungen und hauptsächlich Organhalluzinationen). Er kam zur Behandlung nach Konstantinopel und hatte einige typische Malariaanfälle, die nach Chininbehandlung aufhörten. Mit ihnen hörte die Geistesstörung auf.

Ein anderer Kranker hatte mehrere Malariaanfälle und zeigte bei seiner Ankunft in Konstantinopel eine depressive Neurasthenie. Die gewöhnliche Behandlung der Neurasthenie gab keinen Erfolg, das Blut zeigte Laveransche Halbmonde. Chinin brachte völlige Heilung.

Die Bevölkerung hat als Erbstück aus alten Zeiten alte Glaubensvorstellungen beibehalten, wohl infolgedessen ist die hysterische und mystische Folie sehr verbreitet, sie fehlt auch nicht unter den Aerzten.

Die allgemeine Paralyse ist sehr häufig in Konstantinopel Alle unsere Paralytiker sind mit.ziemlicher Sicherheit syphilitisch 
gewesen, und wenn sie auch nicht immer die Baylesche Krankheit gehabt haben, so hatten sie doch andere Krankheitserscheinungen Aber der Alkohol bringt bei einem Syphilitischen die Paralyse zum Ausbruch. Die Formen der allgemeinen Paralyse sind auch im Orient sehr verschieden. Depression, Expansion, Halluzination, hauptsächlich des Gesichts, Katatonie, Taboparese etc. Man findet sie häufig gegen das 40. Lebensjahr, doch ist sie auch vor dem 30. nicht selten.

Die Dementia praecox ist ziemlich häufig. Beide Geschlechter und alle Berufe steuern dazu bei. Bei den Studierenden nimmt sie häufiger die Form der Hebephrenie und der Dementia paranoides, bei den Frauen häufiger die der Katatonie an.

Die organischen Krankheiten des Nervensystems sind zahlreich, die Syringomyelie führt Prof. Raschid auf die Kälte des Landes zurück, weshalb sie bei Erzerım und Harput so häufig ist. Man trifft sie schon bei sehr jungen Leuten.

Die primären Myopathien sind ebenfalls häufig bei uns. 